International Mathematical Forum, 2, 2007, no. 63, 3105 - 3113

\title{
Minor Extremal Problems Using Turán Graphs
}

\author{
M. Cera, A. Diánez, P. García-Vázquez \\ Departamento de Matemática Aplicada I \\ Universidad de Sevilla, Sevilla, Spain \\ m.cera@us.es, anadianez@us.es, pgvazquez@us.es
}

\section{J. C. Valenzuela}

Departamento de Matemáticas

Universidad de Cádiz, Cádiz, Spain

jcarlos.valenzuela@uca.es

\begin{abstract}
The extremal number $\operatorname{ex}\left(n ; M K_{p}\right)$ denotes the maximum number of edges of a graph of order $n$ not containing a complete graph $K_{p}$ as a minor. In this paper we find a lower bound for this extremal number in terms of Turán Numbers that improves the only lower bound known up to now and it is best possible for infinitely many values of $n$ and $p$. This lower bound also allows us to prove that every Turán Graph $T_{r}(n)$ is shown to contain $K_{p}$ as a minor for all $n \geq 2 p-2$.
\end{abstract}

Keywords: extremal graph theory, minor complete subgraphs

\section{Introduction}

All graphs in this paper are assumed to be simple (undirected, no loops, no multiple edges) and finite. In our notations we follow the books by Bollobás [1] and by Diestel [5], and all missing definitions can be found there. Let $V(G)$ and $E(G)$ denote the set of vertices and the set of edges of the graph $G$, respectively. $|V(G)|$ is called the order of $G$, and $|E(G)|$ is called the size of $G$. For $S \subset V(G)$, the neighborhood of $S$, denoted by $N_{G}(S)$, is the set of vertices in $V(G) \backslash S$ that are adjacent to some vertex of $S$. If $S=\{v\}$ we put simply $N_{G}(v)$. A complete graph of order $p$, denoted by $K_{p}$, comprises $p$ pairwise adjacent vertices. The complement graph of a graph $G$, denoted 
by $\bar{G}$, is the graph with vertex set $V(\bar{G})=V(G)$ defined in such a way that $x y \in E(\bar{G})$ iff $x y \notin E(G)$. A subset of vertices $U \subset V(G)$ is called a vertex cover of $G$ if every edge of $G$ has at least one vertex in $U$, or equivalently, if $G-U$ has no edges. For any subset $S \subseteq V(G)$, the induced subgraph of $G$ by $S$, denoted by $G[S]$, is the graph with vertex set $S$ whose edges are the edges of $G$ joining vertices of $S$. For any two positive integer $r, n$, with $r \leq n$, the Turán Graph $T_{r}(n)$ is the complete $r$-partite graph of order $n$ with $r$ partite sets as equal as possible. The size of this graph is denoted by $t_{r}(n)$. Given a graph $G$ and an edge $e=x y$ of $G$, by $G / e$ we denote the graph obtained from $G$ by contracting the edge $e$ into vertex $x$, which also becomes adjacent to all the former neighbors of $y$. Formally, $G^{\prime}=G / e$ is a graph with vertex set $V^{\prime}=V(G) \backslash\{y\}$ and such that $N_{G^{\prime}}(z)=N_{G}(z)$, for all $z \in V(G) \backslash\{x\}$ and $N_{G^{\prime}}(x)=N_{G}(x) \cup N_{G}(y)$ (multiple edges, if any, are removed). Figure 1 illustrates an example of an edge-contraction in a graph $G$. We say that $H$ is a minor of $G(H=M G)$ or equivalently, that $G$ is contractible to $H$ if $H$ can be obtained from $G$ by a finite sequence of edge-contractions.
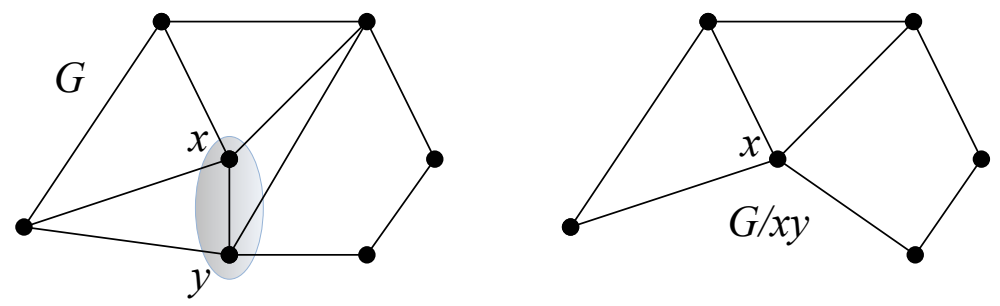

Figure 1: Contracting the edge $x y$ into vertex $x$.

One of the most interesting problems in Extremal Graph Theory consists in studying the extremal function $\operatorname{ex}(n ; \mathcal{F})$, i.e., the maximum possible size of a graph on $n$ vertices free of graphs of the family $\mathcal{F}$. The best known result on this problem, proved in 1941, is due to Turán [16] and it answers the question for the case in which $\mathcal{F}=\left\{K_{p}\right\}$. The solution is given by the number of edges of the Turán Graph $T_{p-1}(n)$. This graph is shown to be the only extremal graph for the problem, that is, the only graph of order $n$ and size ex $\left(n ; K_{p}\right)$ not containing $K_{p}$ as a subgraph. Since then several problems have been proposed involving another families $\mathcal{F}$ of forbidden graphs but many of them are opened yet. In this paper we focus our interest in one of these problems. More precisely, we study the extremal number ex $\left(n ; M K_{p}\right)$, that is, the maximum number of edges of a graph on $n$ vertices not containing $K_{p}$ as a minor. Mader [11] was the first one that proved the existence of a 
constant $c_{p} \leq\left\lceil 2 c_{p-1}\right\rceil$ such that $e x\left(n ; M K_{p}\right) \leq c_{p} n$, for each $p \geq 2$. Later, the same author showed that $c_{p} \leq 8 p \log _{2}(p)$. De la Vega [4] and Thomason [13], by using random graphs, advanced in the study of $c_{p}$ proving that $c_{p} \geq \frac{1}{4} \sqrt{\log _{2}(p)}$. The study was improved by Kostochka [9] who showed that this is the correct order of magnitude of $c_{p}$ and by Thomason [13] who proved that $c_{p} \leq 2.68 p \sqrt{\log _{2}(p)}$. Finally, Thomason [14] completed the problem by showing that $c_{p}=(\alpha+o(1)) t \sqrt{\log t}$. The existence of complete minor has been studied from another points of view (see for instance [10]). More details about extremal functions for graph minors can be found in the survey by Thomason [15].

All the above mentioned works provide us very interesting upper bounds for the extremal number $e x\left(n ; M K_{p}\right)$ in an asymptotical way, i.e., when $p$ is a fixed integer and $n$ is much larger than $p$. Regarding to lower bounds, by considering the graph $G=K_{p-2}+\overline{K_{n-p+2}}$, it is immediate to deduce the following one:

$$
e x\left(n ; M K_{p}\right) \geq(p-2) n-\left(\begin{array}{c}
p-1 \\
2
\end{array}\right) .
$$

However inequality (1) is an equality only for small values of $p$. Dirac [6] proved that $\operatorname{ex}\left(n ; M K_{p}\right)=(p-2) n-\left(\begin{array}{c}p-1 \\ 2\end{array}\right)$ for $p \leq 5$ and Mader [12] showed it for $p \leq 7$. But it only suffices to see the exact value

$$
\operatorname{ex}\left(n ; M K_{8}\right)= \begin{cases}6 n-20, & \text { if } 5 \text { divides } n \\ 6 n-21, & \text { otherwise }\end{cases}
$$

obtained by Jorgensen [7] to check that (1) is not optimum yet.

In this paper we find a new lower bound for the extremal number ex $\left(n ; M K_{p}\right)$ that improves (1) and it is best possible for infinitely many values of $n$ and $p$. This lower bound also allows us to prove that every Turán Graph $T_{r}(n)$ contains $K_{p}$ as a minor for all $n \geq 2 p-2$.

\section{A lower bound for the extremal function}

In this section we find a new lower bound for the extremal number $e x\left(n ; M K_{p}\right)$. First, we need to relate the minimum cardinality of a vertex cover of certain graph with the minimum number of edge-contractions that are necessary in a graph in order to get a complete graph.

Lemma 2.1 Let $G$ be a graph and let $U=\left\{v_{1}, \ldots, v_{p}\right\}$ be a subset of vertices of $G$. Let us denote by $s \leq p$ the minimum cardinality of a vertex cover of 
$\bar{G}[U]$. If $G$ is contractible to a $K_{p}$ with set of vertices $U$, then the minimum number of edge-contractions that are necessary to obtain $K_{p}$ is at least s.

Proof. Let us denote by $H=\bar{G}[U]$. If $H$ has no edges then we are done, because $G[U]=K_{p}, s=0$ and no edge-contractions are necessary. Then assume that $E(H) \neq \emptyset$ and let $W=\left\{w_{1}, \ldots, w_{s}\right\} \subset U$ be a vertex cover of $H$ with minimum cardinality. Suppose that there exists a set of edges $E^{\prime}=\left\{e_{1}, \ldots, e_{r}\right\} \subset E(G)$, with $r<s$, such that the graph obtained from $G$ by contracting these edges contains a complete graph $K_{p}$ with set of vertices $U$. Then there exists at least one vertex $w_{j}$ such that none of the edges of $E^{\prime}$ is contracted in $w_{j}$. But since $G$ is contractible to a $K_{p}$ with set of vertices $U$, then

$$
N_{H}\left(w_{j}\right) \subseteq \bigcup_{\substack{i=1 \\ i \neq j}}^{s} N_{H}\left(w_{i}\right)
$$

Hence $W \backslash\left\{w_{j}\right\}$ is a vertex cover of $H$ with cardinality $s-1$, and this is not possible.

Lemma 2.1 allows us to deduce a sufficient condition in order to show that a graph $G$ is not contractible to a complete graph $K_{p}$, as it is stated in the following result.

Proposition 2.1 Let $n, p$ be positive integers, with $n \geq p$, and let $G$ be a graph on $n$ vertices. If for each subset $U$ of vertices of $G$, the minimum cardinality of a vertex cover of $\bar{G}[U]$ is $s>n-p$, then $G$ does not contain $K_{p}$ as a minor.

Proposition 2.1 permits us to split the region of pairs $(n, p)$, with $n \geq p$, into two parts separated by the curve $n=2 p-3$. On the one hand, there exist Turán Graphs on $n$ vertices not containing $K_{p}$ as a minor, if $p \leq n \leq 2 p-3$. On the other hand, every Turán Graph on $n$ vertices contains $K_{p}$ as a minor, if $n \geq 2 p-2$.

Theorem 2.1 Let $n, p, r$ be positive integers such that $p \leq n \leq 2 p-3$ and $2 \leq r \leq 2 p-n-1$. Then Turán Graph $T_{r}(n)$ is not contractible to $K_{p}$.

Proof. Let us denote by $C_{i}$, with $i \in\{1, \ldots, r\}$, the partite sets of vertices of $T_{r}(n)$. Let $U=\left\{v_{1}, \ldots, v_{p}\right\}$ be any set of vertices of $T_{r}(n)$ and $H$ be the induced subgraph in $\overline{T_{r}(n)}$ by the vertices of $U$; that is, $H=\overline{T_{r}(n)}[U]$. 
Let us denote by $r_{i}=\left|U \cap C_{i}\right|$, for $i=1, \ldots, r$ and consider the set $I=$ $\left\{j \in\{1, \ldots, r\}: r_{j} \geq 1\right\}$.

Observe that $H=\bigcup_{i \in I} K_{r_{i}}$. Then, if we denote by $s$ the minimum cardinality of a vertex cover of $H$, clearly we have

$$
s=\sum_{i \in I}\left(r_{i}-1\right)=\sum_{i \in I} r_{i}-|I|=p-|I| \geq p-r \geq p-(2 p-n-1)>n-p .
$$

Thus, by applying Corollary 2.1, Turán Graph $G=T_{r}(n)$ is not contractible to $K_{p}$.

As an immediate consequence of Theorem 2.1 we deduce a lower bound for the extremal number $\operatorname{ex}\left(n ; M K_{p}\right)$ in terms of the Turán Numbers.

Corollary 2.1 Let $n, p$ be positive integers such that $p \leq n \leq 2 p-3$. Then

$$
e x\left(n ; M K_{p}\right) \geq t_{2 p-n-1}(n) .
$$

Next, our purpose is to show that the pairs of values of $n$ and $p$ described in Theorem 2.1 are the only ones for which there are Turán Graphs not containing $K_{p}$ as a minor.

Theorem 2.2 Let $n, p, r$ be positive integers such that $n \geq 2 p-2$ and $r \geq 2$. Then every Turán Graph $T_{r}(n)$ contains $K_{p}$ as a minor.

Proof. If $r \geq p$ the result clearly holds, hence assume that $2 \leq r \leq p-1$. Let us denote by $C_{i}$, with $i \in\{1, \ldots, r\}$, the partite sets of vertices of $T_{r}(n)$. Let $U$ be a set of vertices of $G$ chosen in such a way that $\left|U \cap C_{i}\right|=\left\lceil\frac{p}{r}\right\rceil$, for $i \in\left\{1, \ldots, p-r\left\lfloor\frac{p}{r}\right\rfloor\right\}$ and $\left|U \cap C_{i}\right|=\left\lfloor\frac{p}{r}\right\rfloor$, for $i \in\left\{p-r\left\lfloor\frac{p}{r}\right\rfloor+1, \ldots, r\right\}$. Clearly $|U|=p$ and the induced subgraph in $T_{r}(n)$ by $U, T_{r}(n)[U]$, is a Turán Graph $T_{r}(p)$. Therefore, if we denote by $H$ the complement graph of $T_{r}(n)[U]$, we deduce that $H$ is a graph on $p$ vertices formed by $r$ disjoint copies of complete graphs. Some of them are copies of $K_{\left\lceil\frac{p}{r}\right\rceil}$ and the rest of them are copies of $K_{\left\lfloor\frac{p}{r}\right\rfloor}$. Thus, the minimum cardinality of a vertex cover of $H$ is

$$
s=p-r \leq p-2=(2 p-2)-p \leq n-p .
$$

Notice that the edges of $H$ are the necessary ones in $T_{r}(n)[U]$ to be a complete graph $K_{p}$. So we need to do some edge-contractions in $T_{r}(n)$ in order to obtain 
a $K_{p}$ with set of vertices $U$. Let $W=\left\{w_{1}, \ldots, w_{s}\right\}$ be a vertex cover of $H$ with minimum cardinality and consider the bipartite graph $B$ whose vertex classes are $W$ and $Z=V\left(T_{r}(n)\right) \backslash U$ defined in such a way that a vertex $w_{i}$ is adjacent to $v_{j}$ in $B$ if $w_{i} v_{j} \in E\left(T_{r}(n)\right)$ (see Figure 2).

If the bipartite graph $B$ has a complete matching $M$ then it suffices to contract in $T_{r}(n)$ the edges of $M$ to obtain a new graph $T_{r}(n) / M$ containing a copy of $K_{p}$ with set of vertices $U$ (see Figure 2). Let us see that there exists a complete matching in $B$. For that, we will use the Hall's Condition [8] for complete matching, which says that $\left|N_{B}(A)\right| \geq|A|$ for all $A \subseteq W$ is a sufficient and necessary condition for the existence of a complete matching in $B$.
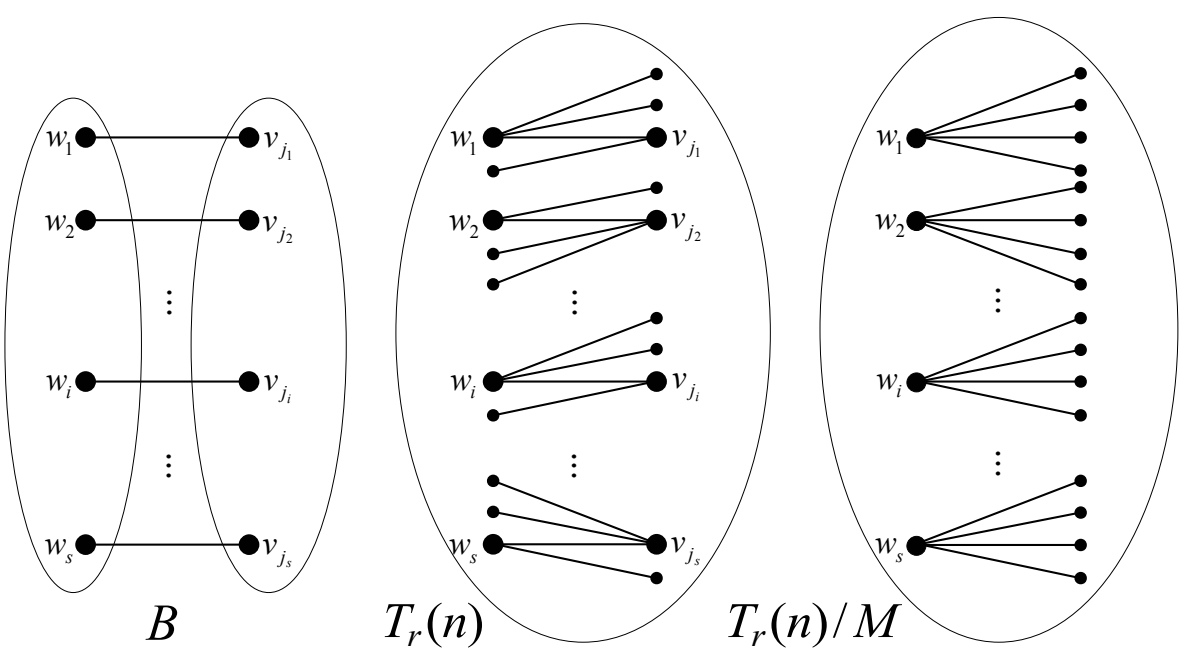

Figure 2: A complete matching in $B$ implies the existence of a minor of $K_{p}$ in $T_{r}(n)$.

Let $A$ be a subset of $W$. If there exist $w, \widetilde{w} \in A$ such that $w \in U \cap C_{j}$ and $\widetilde{w} \in U \cap C_{k}$, with $j \neq k$, then $N(A)=Z$, because $N(\{w\})=Z \backslash\left(Z \cap C_{j}\right)$ and $N(\{\widetilde{w}\})=Z \backslash\left(Z \cap C_{k}\right)$. Then $|N(A)|=|Z|=n-p \geq s=|W| \geq|A|$. So we need to prove that $\left|N\left(W \cap C_{j}\right)\right| \geq\left|W \cap C_{j}\right|$, for all $j \in\{1, \ldots, r\}$, i.e., that

$$
\left|W \cap C_{j}\right| \leq \sum_{\substack{i=1 \\ i \neq j}}^{r}\left|Z \cap C_{i}\right|, \text { for all } j \in\{1, \ldots, r\} .
$$

For each fixed $j \in\{1, \ldots, r\}$ we have 


$$
\begin{aligned}
\sum_{\substack{i=1 \\
i \neq j}}^{r}\left|Z \cap C_{i}\right| & =n-p-\left|Z \cap C_{j}\right| \\
& =\left(n-p-\left|C_{j}\right|+1\right)+\left(\left|C_{j}\right|-1-\left|Z \cap C_{j}\right|\right) \\
& =\left(n-p-\left|C_{j}\right|+1\right)+\left|W \cap C_{j}\right| \\
& \geq\left(n-p-\left\lceil\frac{n}{r} \mid+1\right)+\left|W \cap C_{j}\right|\right. \\
& \geq\left(n-p-\left[\frac{n}{2} \mid+1\right)+\left|W \cap C_{j}\right|\right. \\
& \left.=\mid \frac{n-2 p+2}{2}\right\rfloor+\left|W \cap C_{j}\right| \\
& \geq\left|W \cap C_{j}\right|,
\end{aligned}
$$

because $n \geq 2 p-2$. Thus, by applying the Hall's Condition, there exists a complete matching in the graph $B$ and therefore, Turán's Graph $T_{r}(n)$ contains $K_{p}$ as a minor.

\section{Conclusions and open problems}

In this work we have found a relationship between Turán's Problem and one of its extensions. We have gotten a new lower bound that approaches the exact solution of the problem for infinitely many related values of $n$ and $p$.

Indeed, we think that if $p \leq n \leq g(p)$, with $g(p) \approx 2 p-6$, it seems impossible to find out a lower bound that improves the new one obtained in terms of Turán Numbers. Furthermore this bound is optimum for $6 \leq p \leq 8$ (see [7] and [12]) and if $\left\lceil\frac{5 n+9}{8}\right\rceil \leq p \leq n$ (see [2, 3]). Hence, an interesting open problem would be to prove the following conjecture.

Conjecture 3.1 Let $n, p$ be positive integers such that $p \leq n \leq 2 p-6$. Then

$$
e x\left(n ; M K_{p}\right)=t_{2 p-n-1}(n) .
$$

\section{Acknowledgments}

This research was partially supported by the Ministry of Education and Science, Spain, and the European Regional Development Fund (ERDF) under project MTM2005-08990-C02-02, and by the project of Andalusian Government, Spain EXC/2006/FQM164-1649. 


\section{References}

[1] B. Bollobás, Extremal Graph Theory, Academic Press, London, 1978.

[2] M. Cera, A. Diánez, P. García-Vázquez, and J.C. Valenzuela, Minor clique free extremal graphs, Ars Combinatoria 73, (2004) 153-162.

[3] M. Cera, A. Diánez, P. García-Vázquez, and J.C. Valenzuela, Graphs without minor complete subgraphs, Discrete Math. 307, (2007) 1276-1284.

[4] W.F. De la Vega, On the maximum density of graphs which have no subcontraction to $K_{s}$, Discrete Math. 46 (1983) 109-110.

[5] R. Diestel, Graph Theory (Springer Verlag, New York, 2000).

[6] G.A. Dirac, Homomorphism theorems for graphs, Math. Ann. 153, (1964) 69-80.

[7] Leif K. Jorgensen, Contractions to $K_{8}$, J. Graph Theory 18, (1994) 431448.

[8] P. Hall, On representatives of subsets, J. London Math. Soc. 10, (1935) $26-30$.

[9] A.V. Kostochka, Lower bound of the Hadwiger number of graphs by their average degree, Combinatorica 4, (1984) 307-316.

[10] D. Kühn, Forcing a $K_{r}$ minor by high external connectivity, J. Graph Theory 39 (2002), 241-264.

[11] W. Mader, Homomorphieeigenschaften und mittlere Kantendichte von Graphen, Math. Annalen 174 (1967) 265-268.

[12] W. Mader, Homomorphiesätze für Graphen, Math. Ann. 178 (1968) 154168.

[13] A. Thomason, An extremal function for contractions of graphs, Math. Proc. Cambridge Phil. Soc. 95, (1984) 261-265.

[14] A. Thomason, The extremal function for complete minors, J. Combin. Theory Ser. B 81 (2001), 318-338.

[15] A. Thomason, Extremal functions for graph minors, More sets, graphs and numbers, Bolyai Soc. Math. Stud. 15 (2006), 359-380. 
[16] P. Turán, Eine extremalaufgabe aus der graphentheorie, Mat. Fiz. Lapok 48 (1941) 436-452.

Received: May 24, 2007 